\title{
Temporary Dental Restoration
}

National Cancer Institute

\section{Source}

National Cancer Institute. Temporary Dental Restoration. NCI Thesaurus. Code C60720.

Reconstruction of a tooth or part of a tooth which is designed to seal the tooth and maintain its position until a permanent restoration is done. 